\title{
Flora vascular de uma área de campo úmido em Analândia, estado de São Paulo
}

\author{
Vascular flora of moist grassland area in Analândia, state of São Paulo
}

Naiara Lopes de Sousa ${ }^{1,2}$ \& Julio Antonio Lombardi ${ }^{1}$

\begin{abstract}
Resumo
A fitofisionomia de campo úmido integra-se ao bioma Cerrado quando próximo a nascentes e cursos d'água, e emáreas com lençol freático superficial. Devido às condições edáficas particulares que restringem e selecionam as espécies, esse ambiente apresenta uma flora bastante típica. O presente estudo objetivou listar as espécies de plantas vasculares registradas em área de campo úmido situada na Fazenda Pedra Vermelha, município de Analândia, estado de São Paulo, e comparar os resultados com outros de áreas campestres alagadas no Brasil. A área amostrada possui 5 ha e está situada entre as coordenadas 22 7'50"-8' ' 'S e 47046'40"-53"O, a 760-770 m de altitude. Foram realizados 18 expedições mensais de coleta, registrando-se 178 espécies em 121 gêneros e 62 famílias. Asteraceae (19 espécies), Cyperaceae (15), Poaceae (14), Melastomataceae (13), Xyridaceae e Eriocaulaceae (8) mostraram-se mais diversas. Uma provável espécie nova para Cyperaceae foi diagnosticada e sete espécies encontram-se listadas como em risco de extinção. A similaridade florística entre o campo úmido amostrado e as áreas usadas para comparação apresentou-se baixa. Os resultados obtidos comprovam uma riqueza florística significativa para o campo úmido da Fazenda Pedra Vermelha. Palavras-chave: campos úmidos, Cerrado, estado de São Paulo, estrato herbáceo, florística.

Abstract

The moist grassland phyto-physiognomy associates with the Cerrado biome near springs and streams, and in areas with shallow groundwater. This vegetation has a very typical flora, due the particular soil conditions that restrict and select species. The main objective of this study was to record the species of vascular plants in moist grassland in Fazenda Pedra Vermelha, at Analândia municipality, São Paulo, and to compare the floristic composition with other wetlands areas in Brazil. The area has 5ha and is located between the coordinates 22 07'50"-08'01'S and $47^{\circ} 46^{\prime} 40^{\prime \prime}-53$ ' $\mathrm{O}$, and at altitudes ranging from 760 to $770 \mathrm{~m}$. The floristic survey was carried along 18 months. A total of 178 species, 121 genera and 62 families were registered in the area. Asteraceae (19 species), Cyperaceae (15), Poaceae (14) Melastomaceae (13) Xyridaceae and Eriocaulaceae (8) were the richest families. A probable new species of Cyperaceae was diagnosed and seven endangered species were found. The floristic similarity between this sampled moist grassland and the compared areas was low. The results show a significant floristic richness of the moist grassland at the Fazenda Pedra Vermelha.
\end{abstract}

Key words: moist grassland, Cerrado, São Paulo state, herbaceous layer, floristic.

\section{Introdução}

Considerado um complexo vegetacional heterogêneo (Rizzini 1963), o bioma Cerrado é constituído basicamente por fisionomias vinculadas a solos bem drenados. Contudo, são vistas formações associadas a terrenos com excedente hídrico superficial, correspondendo aos ecossistemas de áreas úmidas, tais como os campos úmidos (Ribeiro \& Walter 2008).
As áreas campestres úmidas normalmente ocorrem em regiões planas próximas a nascentes, cursos d'água, em fundo de vales e em áreas com lençol freático superficial, geralmente entre matas de galeria e o cerrado sensu stricto, atuando como uma área de transição entre essas fitofisionomias (Eiten 1983; Tannus \& Assis 2004; Munhoz \& Felfili 2006b, 2007; Ribeiro \& Walter 2008; Eugênio et al. 2011).

\footnotetext{
${ }^{1}$ Universidade Estadual Paulista - UNESP, Depto. Botânica, Instituto de Biociências de Rio Claro, Av. 24-A 1515, 13506-900, Rio Claro, SP, Brasil.

${ }^{2}$ Autor para correspondência: naiaralsbio@gmail.com
} 
Devido às condições edáficas particulares dos campos úmidos, esses ambientes possuem flora bastante típica (Munhoz \& Felfili 2008). A absorção de nutrientes se torna comprometida pelo estado de alagamento do solo, o que seleciona e restringe o número de espécies com adaptações morfofisiológicas tolerantes a essas condições abióticas e que conseguem persistir na comunidade (Ivanauskas et al. 1997; Amorim \& Batalha 2006).

Apesar de serem considerados sistemas conservatórios de água, essas fisionomias atualmente apresentam-se ameaçadas, principalmente no que diz respeito à preservação dos aquíferos (Reatto et al. 1998). Devido à prática de drenagem para o uso agrícola, o nível dos lençóis freáticos vem sofrendo rebaixamento, sendo contaminados pelo uso indiscriminado de pesticidas e fertilizantes, e pelo despejo de esgotos (Meirelles et al. 2006), além dos distúrbios causados na vegetação original devido à significativa presença de plantas invasoras, causando a diminuição da riqueza florísticas nos campos úmidos (Batalha 1997).

Ainda que Kier et al. (2005) tenham afirmado que as áreas úmidas estão entre as fitofisionomias menos inventariadas do mundo, nos últimos anos, observa-se um interesse e consequente aumento dos estudos sobre a composição da vegetação (Batalha 1997; Araújo et al. 2002; Tannus \& Assis 2004; Linsingen et al. 2006; Munhoz \& Felfili 2006a, 2007; Rezende 2007; Tannus 2007; Amaral 2008), motivados pela grande riqueza florística, bem como pela ocorrência restrita destas formações quando associadas ao Cerrado, e pelas transformações que a paisagem vem passando devido às intensas ações antrópicas.

Com isso, essa contribuição tem como objetivo ampliar o conhecimento sobre as áreas campestres alagadas através do inventário das espécies ocorrentes no campo úmido situado na Fazenda Pedra Vermelha, Analândia, estado de São Paulo, e analisar comparativamente outras áreas de campos úmidos inventariados no Brasil.

\section{Materiais e Métodos}

O campo úmido estudado compreende 5 hectares localizados na Fazenda Pedra Vermelha (FPV) no município de Analândia, estado de São Paulo, entre as coordenadas 2207'50'-8' 1 'S e 47046'40"-53"O, a 760-770 m de altitude sobre o nível do mar. O clima da região é do tipo tropical de altitude - Cwa que, segundo Köppen possui temperaturas médias entre $18^{\circ}$ e $23^{\circ} \mathrm{C}$, com verões quentes e chuvosos e uma estação seca no inverno, podendo ocorrer geadas esporádicas. Baseado na classificação da Empresa Brasileira de Pesquisa Agropecuária - Embrapa (2006) o solo da área é tido como organossolo por apresentar coloração escura, acúmulo de restos vegetais em decomposição, e por encontrar-se associado a superfície com pouca drenagem, com consequente alagamento.

Situado na porção sudoeste da propriedade, onde há um predomínio de vegetação de Cerrado, o campo úmido encontra-se delimitado a nordeste por plantação de eucalipto, e pela presença de matas de galeria inundáveis no entorno dos ribeirões do Feijão e Quebra Canela a sudeste e a oeste, respectivamente, os quais, juntamente com o lençol freático superficial, influenciam diretamente no nível de umidade do solo, principalmente na estação chuvosa, onde o alagamento apresenta-se maior quando comparado com a estação seca (Fig. 1).

Para o levantamento florístico no campo úmido da Fazenda Pedra Vermelha foram realizadas 18 expedições de coleta mensais, através de caminhadas aleatórias ao longo da área a partir da estrada de acesso (Filgueiras et al. 1994).

O material botânico foi preparado segundo técnicas usuais de herborização, conforme proposto por Fidalgo \& Bononi (1984). As exsicatas foram incorporadas ao Herbário Rioclarense (HRCB) do Instituto de Biociências de Rio Claro da Universidade Estadual Paulista "Júlio de Mesquita Filho".

As identificações taxonômicas foram feitas por meio de literatura especializada e chaves de identificação, bem como por comparação mediante consultas a herbários, e por determinação de especialistas em diferentes grupos taxonômicos. A classificação dos espécimes segue o modelo proposto pelo Angiosperm Phylogeny Group (APG) III (2009) para o reconhecimento das famílias de angiospermas, enquanto que aquela das Lycophytas e Monilophytas estão baseadas, respectivamente, em Kramer \& Tryon (1990) e Smith et al. (2006). As espécies foram classificadas quanto aos tipos de hábitos (herbáceo, subarbustivo, arbustivo, arbóreo e trepador) como definidos em Gonçalves \& Lorenzi (2011).

Quanto á similaridade florística, utilizou-se o índice de Sorensen para comparar as diferentes áreas de campo úmido já inventariadas (MuellerDombois \& Ellemberg 1974). A sinonimia dos táxons foi conferida através de Prado et al. (2015) e BFG (2015). 




Figura 1 - Mapa da localização do campo úmido em relação à Fazenda Pedra Vermelha, situada no município de Analândia, São Paulo.

Figure 1 - Location's map of the moist grassland relative to Fazenda Pedra Vermelha, located in the municipality of Analândia, Sao Paulo.

\section{Resultados e Discussão}

Foram encontradas 178 espécies (Tab. 1) para o campo úmido da FPV, as quais estão distribuídas em 121 gêneros e 62 famílias, sendo $85 \%$ delas pertencentes ao grupo das Angiospermas (53 famílias), $13 \%$ ao das Monilófitas (8) e $2 \%$ das Licófitas (1).

Famílias como Asteraceae (19 espécies), Cyperaceae (15), Poaceae (14), Melastomataceae (13), Xyridaceae e Eriocaulaceae (8) apresentaramse com o maior número de espécies, totalizando $43 \%$ da riqueza registrada. As mesmas têm sido apontadas como as mais representativas nos estudos sobre a flora de áreas alagadas no
Brasil, apesar de não estarem restritas a esse tipo de ambiente, visto que Asteraceae, Poaceae e Melastomataceae também estão entre aquelas mais diversas para o bioma Cerrado (Mendonça et al. 2008). Os gêneros de grande importância quantitativa são representados por Xyris (8 espécies), Rhynchospora (7), Utricularia (6) e Ocotea (4). Destes, Xyris e Rhynchospora também se destacaram nos levantamentos florísticos de regiões campestres úmidas.

A cobertura herbácea densa da área está representada por $63,5 \%$ das espécies coletadas. Acima deste estrato observam-se indivíduos arbustivos e subarbustivos que, juntos, equivalem 
Tabela 1 - Lista das espécies coletadas no campo úmido da Fazenda Pedra Vermelha, Analândia, São Paulo indicando para cada espécie a família a qual pertence, o hábito e o número do coletor.

Table 1 - List of species collected in moist grassland in Fazenda Pedra Vermelha, Analândia, São Paulo indicating the species, the family, the habit and the number of collector.

Família / Espécie Hábito Número do coletor

\section{ALISMATACEAE}

Echinodorus paniculatus Micheli

Herbáceo

N.L.S. 303

APIACEAE

Eryngium ebracteatum Lam.

Herbáceo

N.L.S. 306

APOCYNACEAE

Mandevilla scabra (Hoffmanns. ex Roem. \& Schult.) K.Schum.

Trepador

N.L.S. 298

Mandevilla tenuifolia (J.C.Mikan) Woodson

Herbáceo

N.L.S. 289

Oxypetalum pachygynum Decne.

Trepador

N.L.S. 341

Peplonia axillaris (Vell.) Fontella \& Rapini

Trepador

J.A.L. 9314

\section{AQUIFOLIACEAE}

Ilex affinis Gardner

Ilex brasiliensis (Spreng.) Loes.

Arbóreo

N.L.S. 235

ARACEAE

Xanthosoma striatipes (K.Koch \& C.D.Bouché) Madison

Arbóreo

N.L.S. 253

\section{ASTERACEAE}

Achyrocline alata (Kunth) DC.

Herbáceo

N.L.S. 304

Acilepidopsis echitifolia (Mart. ex DC.) H.Rob.

Ageratum conyzoides L.

Herbáceo

J.A.L. 9245

Ageratum fastigiatum (Gardner) R.M.King \& H.Rob.

Herbáceo

J.A.L. 9210

Baccharis dracunculifolia DC.

Herbáceo

J.A.L. 9273

Arbustivo

J.A.L. 9291

Baccharis junciformis DC.

Arbustivo

N.L.S. 320

Baccharis singularis (Vell.) G.M.Barroso

Herbáceo

J.A.L. 9222

Chromolaena odorata (L.) R.M.King \& H.Rob.

Arbustivo

J.A.L. 9199

Clibadium armani (Balb.) Sch.Bip. ex O.E.Schulz

Arbustivo

J.A.L. 9201

Elephantopus palustris Gardner

Arbustivo

N.L.S. 249

Emilia sonchifolia (L.) DC. ex Wight

Herbáceo

J.A.L. 9246

Gochnatia pulchra Cabrera

Herbáceo

J.A.L. 9254

Heterocondylus cf. pumilus (Gardner) R.M.King \& H.Rob.

Arbustivo

J.A.L. 9265

Heterocondylus vitalbae (DC.) R.M.King \& H.Rob.

Herbáceo

N.L.S. 273

Jungia floribunda Less.

Arbustivo

J.A.L. 9251

Lessingianthus glabratus (Less.) H.Rob.

Herbáceo

J.A.L. 9264

Mikania micrantha Kunth

Herbáceo

J.A.L. 9197

Verbesina sordescens DC.

Trepador

J.A.L. 9261

Vernonanthura cf. westiniana (Less.) H.Rob.

Arbustivo

J.A.L. 9255

BEGONIACEAE

Begonia cucullata Willd.

Arbustivo

J.A.L. 9198

BIGNONIACEAE 


\section{Família / Espécie \\ Jacaranda caroba (Vell.) DC. \\ BLECHNACEAE \\ Blechnum brasiliense Desv. \\ Blechnum schomburgkii (Klotzsch) C.Chr.}

CAMPANULACEAE

Lobelia exaltata Pohl

Siphocampylus sulfureus E. Wimm.

\section{CARDIOPTERIDACEAE}

Citronella gongonha (Mart.) R.A.Howard

\section{CLUSICACEAE}

Clusia criuva Cambess.

\section{COMMELINACEAE}

Commelina diffusa Burm.f.

\section{CYATHEACEAE}

Cyathea atrovirens (Langsd. \& Fisch.) Domin

Cyathea delgadii Sternb.

\section{CYPERACEAE}

Cyperus cf. distans L.

Cyperus haspan $\mathrm{L}$.

Cyperus cf. luzulae (L.) Retz.

Eleocharis minima Kunth

Eleocharis sp. 1

Rhynchospora albiceps Kunth

Rhynchospora corymbosa (L.) Britton

Rhynchospora emaciata (Nees) Boeckeler

Rhynchospora globosa (Kunth) Roem. \& Schult.

Rhynchospora aff. marisculus Lindl. \& Nees

Rhynchospora aff. rugosa (Vahl) Gale

Scleria latifolia Sw.

Scleria scabra Willd.

Sp. nov. ined.

\section{DIOSCOREACEAE}

Dioscorea hassleriana Chodat

\section{DROSERACEAE}

Drosera communis A.St.-Hil.

\section{ERICACEAE}

Agarista chlorantha (Cham.) G.Don

Gaylussacia brasiliensis (Spreng.) Meisn.

Gaylussacia pseudogaultheria Cham. \& Schltdl.

ERIOCAULACEAE

Hábito

Número do coletor

Arbustivo

N.L.S. 347

Herbáceo

J.A.L. 9321

Herbáceo

J.A.L. 9290

Herbáceo

J.A.L. 9208

Herbáceo

N.L.S. 297

Arbóreo

N.L.S. 236

Arbóreo

N.L.S. 299

Herbáceo

J.A.L. 9220

Herbáceo

J.A.L. 9214

Herbáceo

J.A.L. 9213

Herbáceo

N.L.S. 315

Herbáceo

J.A.L. 9206

Herbáceo

J.A.L. 9293

Herbáceo

N.L.S. 338

Herbáceo

N.L.S. 337

Herbáceo

N.L.S. 263

Herbáceo

J.A.L. 9297

Herbáceo

N.L.S. 319

Herbáceo

N.L.S. 264

Herbáceo

N.L.S. 33

Herbáceo

N.L.S. 238

Herbáceo

J.A.L. 9318

Herbáceo

J.A.L. 9250

Herbáceo

J.A.L. 9275

Trepador

J.A.L. 9217

Herbáceo

N.L.S. 312

Arbustivo

N.L.S. 259

Arbustivo

J.A.L. 9278

Arbustivo

N.L.S. 246

Herbáceo

N.L.S. 305 


\begin{tabular}{|c|c|c|}
\hline Família / Espécie & Hábito & Número do coletor \\
\hline Comanthera xeranthemoides (Bong.) L.R.Parra \& A.M.Giuletti & Herbáceo & N.L.S. 262 \\
\hline Eriocaulon elichrysoides Bong. & Herbáceo & N.L.S. 255 \\
\hline Paepalanthus flaccidus (Bong.) Kunth & Herbáceo & J.A.L. 9232 \\
\hline Paepalanthus aff. planifolius (Bong.) Körn. & Herbáceo & N.L.S. 358 \\
\hline Syngonanthus caulescens (Poir.) Ruhland & Herbáceo & N.L.S. 245 \\
\hline Syngonanthus densiflorus (Körn.) Ruhland & Herbáceo & J.A.L. 9234 \\
\hline Syngonanthus helminthorrhizus (Mart. ex Körn.) Ruhland & Herbáceo & J.A.L. 9233 \\
\hline \multicolumn{3}{|l|}{ ERYTHROXYLACEAE } \\
\hline Erythroxylum cuneifolium (Mart.) O.E.Schulz & Arbustivo & N.L.S. 327 \\
\hline \multicolumn{3}{|l|}{ EUPHORBIACEAE } \\
\hline Croton cf. gracilipes Baill. & Arbóreo & J.A.L. 9215 \\
\hline \multicolumn{3}{|l|}{ FABACEAE } \\
\hline Aeschynomene sensitiva $\mathrm{Sw}$. & Herbáceo & J.A.L. 9316 \\
\hline Chamaecrista cathartica (Mart.) H.S.Irwin \& Barneby & Arbustivo & N.L.S. 342 \\
\hline Chamaecrista rotundifolia (Pers.) Greene & Herbáceo & N.L.S. 318 \\
\hline Senna pendula (Humb. \& Bompl. ex Willd. ) H.S. Irwin \& Barneby & Arbustivo & J.A.L. 9306 \\
\hline \multicolumn{3}{|l|}{ GENTIANACEAE } \\
\hline Chelonanthus alatus (Aubl.) Pulle & Herbáceo & J.A.L. 9240 \\
\hline Helia oblongifolia Mart. & Herbáceo & N.L.S. 284 \\
\hline Schultesia aptera Cham. & Herbáceo & J.A.L. 9239 \\
\hline Schultesia gracilis Mart. & Herbáceo & J.A.L. 9284 \\
\hline \multicolumn{3}{|l|}{ GESNERIACEAE } \\
\hline Sinningia elatior (Kunth) Chautems & Herbáceo & N.L.S. 279 \\
\hline \multicolumn{3}{|l|}{ GLEICHENIACEAE } \\
\hline Dicranopteris flexuosa (Schrad.) Underw. & Herbáceo & J.A.L. 9209 \\
\hline \multicolumn{3}{|l|}{ HYPERICACEAE } \\
\hline Hypericum brasiliense Choisy & Herbáceo & J.A.L. 9307 \\
\hline \multicolumn{3}{|l|}{ IRIDACEAE } \\
\hline Sisyrinchium vaginatum Spreng. & Herbáceo & N.L.S. 242 \\
\hline Iridaceae Indeterminada 1 & Herbáceo & N.L.S. 293 \\
\hline \multicolumn{3}{|l|}{ LAMIACEAE } \\
\hline Hyptis caespitosa A.St.-Hil. ex Benth. & Herbáceo & N.L.S. 336 \\
\hline Hyptis pulchella Briq. & Herbáceo & N.L.S. 333 \\
\hline Hyptis sp. 1 & Arbustivo & J.A.L. 9272 \\
\hline \multicolumn{3}{|l|}{ LAURACEAE } \\
\hline Ocotea lancifolia (Schott) Mez & Arbóreo & N.L.S. 354 \\
\hline Ocotea pulchella (Nees \& Mart.) Mez & Arbóreo & N.L.S. 280 \\
\hline Ocotea tristis (Nees \& Mart.) Mez & Arbustivo & J.A.L. 9298 \\
\hline Ocotea velloziana (Meisn.) Mez & Arbóreo & N.L.S. 352 \\
\hline Persea venosa Nees \& Mart. & Arbóreo & J.A.L. 9271 \\
\hline LENTIBULARIACEAE & & \\
\hline
\end{tabular}




\begin{tabular}{|c|c|c|}
\hline Família / Espécie & Hábito & Número do coletor \\
\hline Utricularia nana A.St.-Hil. \& Girard & Herbáceo & N.L.S. 314 \\
\hline Utricularia nervosa G.Weber ex Benj. & Herbáceo & N.L.S. 267 \\
\hline Utricularia praelonga A.St.-Hil. \& Girard & Herbáceo & N.L.S. 272 \\
\hline Utricularia tricolor A.St.-Hil. & Herbáceo & N.L.S. 269 \\
\hline Utricularia triloba Benj. & Herbáceo & N.L.S. 326 \\
\hline Utricularia sp.1 & Herbáceo & N.L.S. 286 \\
\hline \multicolumn{3}{|l|}{ LORANTHACEAE } \\
\hline Struthanthus marginatus (Desr.) Blume & Arbustivo & N.L.S. 349 \\
\hline \multicolumn{3}{|l|}{ LYCOPODIACEAE } \\
\hline Palhinhaea cernua (L.) Franco \& Vasc. & Herbáceo & N.L.S. 344 \\
\hline \multicolumn{3}{|l|}{ LYGODIACEAE } \\
\hline Lygodium volubile $\mathrm{Sw}$. & Trepador & J.A.L. 9203 \\
\hline \multicolumn{3}{|l|}{ MALPIGHIACEAE } \\
\hline Byrsonima intermedia A.Juss. & Arbustivo & J.A.L. 9302 \\
\hline Heteropterys umbellata A.Juss. & Arbustivo & N.L.S. 288 \\
\hline \multicolumn{3}{|l|}{ MALVACEAE } \\
\hline Byttneria palustris Cristóbal & Subarbustivo & N.L.S. 292 \\
\hline \multicolumn{3}{|l|}{ MELASTOMATACEAE } \\
\hline Acisanthera quadrata Pers. & Subarbustivo & N.L.S. 290 \\
\hline Cambessedesia hilariana (Kunth) DC. & Herbáceo & N.L.S. 283 \\
\hline Leandra polystachya (Naudin) Cogn. & Arbustivo & J.A.L. 9224 \\
\hline Macairea radula (Bonpl.) DC. & Arbustivo & J.A.L. 9228 \\
\hline Miconia chamissois Naudin & Arbóreo & N.L.S. 350 \\
\hline Miconia theizans (Bonpl.) Cogn. & Arbustivo & J.A.L. 9252 \\
\hline Microlepis oleifolia (DC.) Triana & Arbustivo & J.A.L. 9223 \\
\hline Rhynchanthera ursina Naudin & Arbustivo & J.A.L. 9229 \\
\hline Rhynchanthera verbenoides Cham. & Herbáceo & J.A.L. 9281 \\
\hline Tibouchina gracilis (Bonpl.) Cogn. & Herbáceo & J.A.L. 9309 \\
\hline Tibouchina herbacea (DC.) Cogn. & Herbáceo & J.A.L. 9226 \\
\hline Tibouchina ursina (Cham.) Cogn. & Arbustivo & J.A.L. 9227 \\
\hline Trembleya parviflora (D.Don) Cogn. & Arbustivo & N.L.S. 346 \\
\hline \multicolumn{3}{|l|}{ MELIACEAE } \\
\hline Guarea macrophylla Vahl & Arbóreo & N.L.S. 248 \\
\hline \multicolumn{3}{|l|}{ MYRTACEAE (Sobra, M. - UFSJ) } \\
\hline Myrcia hartwegiana (O.Berg) Kiaersk. & Arbóreo & J.A.L. 9267 \\
\hline Myrcia laruotteana Cambess. & Arbóreo & N.L.S. 265 \\
\hline \multicolumn{3}{|l|}{ OCHNACEAE } \\
\hline Ouratea spectabilis (Mart.) Engl. & Arbustivo & J.A.L. 9304 \\
\hline Sauvagesia racemosa A.St.-Hil. & Herbáceo & N.L.S. 300 \\
\hline \multicolumn{3}{|l|}{ ONAGRACEAE } \\
\hline Ludwigia nervosa (Poir.) H.Hara & Arbustivo & J.A.L. 9230 \\
\hline
\end{tabular}




\begin{tabular}{l} 
Família / Espécie \\
\hline Ludwigia tomentosa (Cambess.) H.H \\
ORCHIDACEAE \\
Cleistes aff. montana Gardner \\
Ionopsis utricularioides (Sw.) Lindl. \\
Prescottia aff. oligantha (Sw.) Lindl. \\
Rodriguezia decora (Lem.) Rchb.f.
\end{tabular}

OSMUNDACEAE

Osmunda regalis $\mathrm{L}$.

Osmundastrum cinnamomeum (L.) C.Presl

PHYLLANTHACEAE

Phyllanthus stipulatus (Raf.) G.L.Webster

PIPERACEAE

Piper fuligineum (Kunth) Steud.

POACEAE (Viana, P. - MPEG)

Andropogon bicornis L.

Andropogon virgatus Desv.

Andropogon sp. 1

Anthaenantia lanata (Kunth) Benth.

Dichanthelium surrectum (Chase ex Zuloaga \& Morrone) Zuloaga

Eriochrysis cayennensis P.Beauv.

Eriochrysis holcoides (Nees) Kuhlm.

Luziola bahiensis (Steud.) Hitchc.

Otachyrium versicolor (Döll) Henrard

Paspalum cordatum Hack.

Saccharum asperum (Nees) Steud.

Saccharum villosum Steud.

Trichanthecium parvifolium (Lam.) Zuloaga \& Morrone

Poaceae Indeterminada 1

POLYGALACEAE

Polygala sabulosa A.W.Benn.

POLYGONACEAE

Polygonum meisnerianum Cham. \& Schltdl.

Polygonum rubricaule Cham.

POLYPODIACEAE

Pleopeltis pleopeltifolia (Raddi) Alston

Serpocaulon vacillans (Link) A.R.Sm.

\section{PRIMULACEAE}

Cybianthus densicomus Mart.

Myrsine guianensis (Aubl.) Kuntze

PTERIDACEAE

Hábito

Herbáceo

Herbáceo

Herbáceo

Herbáceo

Herbáceo

Herbáceo

Herbáceo

Arbustivo

Herbáceo

Herbáceo

Herbáceo

Herbáceo

Herbáceo

Herbáceo

Herbáceo

Herbáceo

Herbáceo

Herbáceo

Herbáceo

Herbáceo

Herbáceo

Herbáceo

Herbáceo

Herbáceo

Herbáceo

Herbáceo

Herbáceo

Arbustivo

Arbóreo
N.L.S. 234

N.L.S. 268

N.L.S. 260

N.L.S. 351

N.L.S. 252

J.A.L. 9320

J.A.L. 9313

N.L.S. 296

J.A.L. 9308

J.A.L. 9200

N.L.S. 281

N.L.S. 282

J.A.L. 9207

J.A.L. 9310

J.A.L. 9247

J.A.L. 9315

N.L.S. 325

N.L.S. 321

N.L.S. 241

J.A.L. 9204

J.A.L. 9216

N.L.S. 339

N.L.S. 243

J.A.L. 9305

J.A.L. 9303

N.L.S. 356

N.L.S. 239

J.A.L. 9317

N.L.S. 322 
Família / Espécie

RHAMNACEAE

Rhamnus sphaerosperma $\mathrm{Sw}$.

ROSACEAE

Prunus myrtifolia (L.) Urb.

RUBIACEAE

Borreria multiflora (DC.) Bacigalupo \& E.L.Cabral

Coccocypselum aureum (Spreng.) Cham. \& Schltdl.

Galianthe valerianoides (Cham. \& Schltdl.) E.L.Cabral

Galium equisetoides (Cham. \& Schltdl.) Standl.

Galium hypocarpium (L.) Endl. ex Griseb.

Posoqueria latifolia (Rudge) Schult.

Psychotria tenerior (Cham.) Mull.Arg.

\section{SIPARUNACEAE}

Siparuna brasiliensis (Spreng.) A.DC.

SMILACACEAE

Smilax polyantha Griseb.

SOLANACEAE

Schwenckia curviflora Benth.

Solanum paniculatum $\mathrm{L}$.

SYMPLOCACEAE

Symplocos sp. 1

\section{THELYPTERIDACEAE}

Thelypteris rivularioides (Fée) Abbiatti

\section{THYMELAEACEAE}

Daphnopsis racemosa Griseb.

\section{URTICACEAE}

Cecropia pachystachya Trécul

\section{WINTERACEAE}

Drimys brasiliensis Miers

\section{XYRIDACEAE}

Xyris fallax Malme

Xyris laxifolia Mart.

Xyris rigida Kunth

Xyris savanensis Miq.

Xyris schizachne Mart.

Xyris stenocephala Malme

Xyris tenella Kunth

Xyris tortula Mart.

\section{Hábito}

Arbóreo

N.L.S. 244

Arbóreo

N.L.S. 257

Herbáceo

J.A.L. 9253

Herbáceo

Herbáceo

Herbáceo

Herbáceo

Arbóreo

Arbustivo

Arbustivo

Trepadeira

N.L.S. 270

Herbáceo

N.L.S. 311

Arbustivo

N.L.S. 271

Arbóreo

N.L.S. 330

Herbáceo

N.L.S. 323

Arbóreo

N.L.S. 258

Arbóreo

J.A.L. 9202

Arbóreo

J.A.L. 9279

Herbáceo

J.A.L. 9235

Herbáceo

N.L.S. 275

Herbáceo

N.L.S. 254

Herbáceo

N.L.S. 285

Herbáceo

N.L.S. 294

Herbáceo

N.L.S. 277

Herbáceo

N.L.S. 291

Herbáceo 
a $20 \%$, além de indivíduos arbóreos de pequeno porte com $12,5 \%$ dos táxons. A proporção do número de espécies do componente herbáceosubarbustivo em relação ao arbustivo-arbóreo foi de aproximadamente 2:1. Também registrou-se a presença de uma arbustiva hemiparasita da família Loranthaceae, e de espécies de hábito escandente, pertencentes às famílias Apocynaceae, Asteraceae, Dioscoreaceae, Lygodiaceae e Smilacaceae, que corresponde a $4 \%$ das espécies .

Constatou-se a ocorrência de cinco espécies listadas nas categorias de avaliações de risco de extinção para o estado de São Paulo de acordo com Lista Vermelha da Flora de São Paulo (Mamede et al. 2007), incluindo Echinodorus paniculatus, Mandevilla tenuifolia, Rhynchanthera ursina, $R$. verbenoides, e Galium equisetoides; e outras duas para a flora brasileira, baseado na Lista Vermelha da Flora do Brasil (Martinelli \& Moraes 2013): Schwenckia curviflora e Xyris rigida. Entre as principais causas que as enquadram como ameaçadas pode-se citar a fragmentação dos habitats causadas pelas devastações ambientais; o fato de serem espécies típicas do bioma Cerrado o qual é considerado uma das 25 áreas prioritárias para a conservação, os chamados hotspots mundiais (Myers et al. 2000); a sua distribuição restrita; e/ou a ausência de novos registros de coleta, como assim diagnosticado para Schwenckia curviflora. Essa é uma espécie exclusiva de áreas úmidas e brejosas do sul e sudeste do Brasil que nos últimos 30 anos foi registrada em apenas 3 coletas. No estado de São Paulo, a última foi realizada em 1933 (Martinelli \& Moraes 2013), sendo a presente coleta do campo úmido da FPV o quarto e mais recente registro da espécie.

Para o campo úmido da FPV, uma aparente espécie nova não descrita da família Cyperaceae foi registrada (M. Alves, comunicação pessoal). Eriochrysis holcoides aparece como um novo registro para o estado de São Paulo, de acordo com Checklist das Spermatophytas do Estado de São Paulo, (Wanderley et al. 2011) e com BFG (2015).

Esses resultados demonstram para o conhecimento da distribuição das espécies a importância de inventários criteriosos que evidenciam espécies não descritas, em extinção e novos registros, fenômenos particularmente frequentes em florística de áreas negligenciadas como os campos úmidos. Para Amaral (2008), muitas espécies presentes nessas formações, ou sua ocorrência ali, podem estar desconhecidas devido à escassez de informações.
A presença de alguns táxons com habitats de ocorrência restritos podem atuar como indicadora de áreas úmidas, tais como Rhynchospora albiceps, $R$. globosa, R. rugosa e Syngonanthus densiflorus encontradas somente em áreas de campo úmido e em veredas sobre solos hidromórficos (Munhoz et al. 2008; Munhoz \& Felfili 2008). Já outros, como Emilia sonchifolia e Andropogon bicornis, por serem vistas em áreas campestres alagadas antropizadas são consideradas por Guimarães et al. (2002), e Munhoz \& Felfili (2007) como invasoras dessas formações.

A variação sazonal entre uma estação seca e outra chuvosa é um fenômeno característico do bioma Cerrado. Na área amostrada, essa variação climática foi visivelmente observada influenciando a composição florística. A presença de algumas espécies foi registrada somente ao longo do período de estiagem, a exemplo de Eriocaulon elichrysoides, evidenciando que algumas espécies podem brotar em condições de seca, quando há redução do sombreamento e do alagamento do solo, talvez aproveitando a diminuição da competição para se desenvolverem (Munhoz \& Felfili 2008). Por outro lado, outras aparentam ser mais sensíveis a estas variações, como é o caso de Drosera communis, a qual foi observada em um período muito limitado, apenas no mês de janeiro, e representada por um indivíduo. Em ambientes onde as condições ambientais e do solo são variáveis e interferem diretamente no ciclo reprodutivo das espécies que os colonizam, como nas formações do Cerrado, observa-se a relevância e a necessidade de aplicar períodos de amostragem maiores do que um ano nos levantamentos florísticos, buscando uma maior eficiência da coleta de táxons crípticos.

A fisionomia do campo úmido amostrado tem como característica a formação de touceiras emaranhadas de espécies com folhas filiformes ou estreitas, pertencentes principalmente às famílias Cyperaceae, Poaceae e Xyridaceae, por entre as quais indivíduos de espécies herbáceassubarbustivas conseguem transpor e expor as inflorescências e/ou infrutescências através de longos escapos. Algumas são capazes de se estabelecerem entre touceiras, no interior dos 'tuneis' formados pelas folhas que se curvam para baixo criando um ambiente com pouca luminosidade e maior umidade. Ao longo da área, indivíduos arbóreos mostraram-se reunidos principalmente em pequenas "ilhas" levemente elevadas em relação ao terreno circundante, onde se observa uma redução no grau de umidade do solo. 
Segundo Guimarães e colaboradores (2002), essa diferença na estrutura e na composição de espécies entre ou dentro de ambientes úmidos pode ser uma consequência das alterações no nível do lenço freático. Em meio ao campo úmido da Fazenda Pedra Vermelha, tal fato tem, possivelmente, permitido a instalação de um número significativo de indivíduos arbóreoarbustivos (12,5\% das espécies registradas).

Em ambientes alagados, o estabelecimento do estrato arbóreo-arbustivo é limitado pelas condições de umidade do solo, que acabam influenciando negativamente a germinação e a permanência de plantas de grande porte (Munhoz \& Felfili 2007; Brito et al. 2008; Ribeiro \& Walter 2008). São relativamente poucas as espécies arbóreas tropicais que desenvolveram mecanismos adaptativos que permitem sua sobrevivência nas condições anaeróbicas causadas pelo alagamento. Em se tratando de áreas de campo limpo úmido, é possível registrar a total ausência de espécies arbóreas, como foi observado para a Fazenda da Máquina, no município de Itirapina, São Paulo (Tannus \& Assis 2004).

Para o campo úmido da FPV, sabe-se que desde a década de 80 a área encontra-se isolada da ação do fogo. Este longo período de ausência de queimadas também pode atuar como um fator favorável à instalação das espécies lenhosas (Oliveira-Filho \& Ratter 2002). No Cerrado, as áreas campestres são mantidas por queimadas frequentes, que são capazes de alterar a estrutura original da vegetação, reduzindo a densidade do estrato arbóreo, mais sensível à presença do fogo (Coutinho 1978; Ratter et al. 1997; Munhoz \& Felfili 2006b).

Além disso, por encontrar-se inserida em uma região de transição entre remanescente de cerrado e de mata de galeria, essas fitofisionomias podem estar exercendo uma forte influência quanto à manutenção e à composição das espécies arbóreas. $\mathrm{O}$ que sugere a ocorrência de uma substituição gradativa de espécies no campo úmido da FPV, visto que o aumento progressivo desses indivíduos supostamente vem causando um sombreamento das herbáceas e um consequente declínio da abundância das mesmas, de forma a permitir a futura instalação sucessional de uma estrutura florestal (Scholes \& Archer 1997) se as condições do solo se alterarem. No campo úmido da FPV, a presença de um gênero predominantemente arbóreo (Ocotea) dentre aqueles com o maior número de espécies registradas pode ser vista como um indício dessa sucessão de estratos.
Tannus (2007) também observou esse fenômeno no campo úmido da Estação Ecológica de Itirapina, e afirmou que a instauração de espécies típicas de florestas paludosas e de galeria, como Miconia chamissois, M. theaezans, Ilex affinis, Drimys brasiliensis, Cecropia pachystachya, Cyathea atrovirens e $C$. delgadii, pode ser considerado um indicativo da fase inicial desse processo.

Diante das possíveis evidências da instalação sucessional de uma estrutura florestal no campo úmido da FPV, salienta-se a necessidade de estudos a longo prazo sobre a dinâmica florística da área a fim de verificar-se, ou não, a presença desta substituição de estratos.

Apesar da sua pequena extensão, a área apresentou um relevante número de espécies coletadas, indicando uma expressiva riqueza florística quando comparada a outros levantamentos em formações campestres alagadas associadas ao bioma Cerrado: 64 espécies em um campo úmido do Parque Estadual do Cerrado, em Jaguariaíva, estado do Paraná (Linsingen et al. 2006); 207 espécies para o campo limpo úmido em Alto Paraíso de Goiás (Munhoz \& Felfili 2006a); 197 (Munhoz \& Felfili 2007) e 299 (Amaral 2008) espécies para a Fazenda Água Limpa no Distrito Federal, 136 espécies em campos limpos úmidos associados a veredas no Parque Estadual de Jalapão em Tocantins (Rezende 2007); 526 espécies em quatro comunidades de veredas em Minas Gerais, no município de Uberlândia (Araújo et al. 2002). No estado de São Paulo, registraram-se para o município de Itirapina 114 espécies em área particular (Tannus \& Assis 2004), 167 espécies na Estação Ecológica de Itirapina (Tannus 2007) e 189 espécies na Unidade de Pesquisa e Desenvolvimento de Itararé (Tannus 2007); e para a Área de Relevante Interesse Ecológico Cerrado Pé-de-Gigante, em Santa Rita do Passa Quatro, listou-se 150 espécies (Batalha 1997).

De acordo com o índice de Sorensen, observou-se uma similaridade florística baixa entre o campo úmido amostrado e as áreas campestres alagadas no Brasil (Tab. 2). Esse resultado é visto mesmo entre localidades pouco distantes, como ocorre entre a Fazenda da Máquina (Tannus \& Assis 2004), a Estação Ecológica de Itirapina (Tannus 2007) e a Fazenda Pedra Vermelha, visto que o município de Itirapina, encontra-se a $23 \mathrm{~km}$ de Analândia. Ainda assim, as composições florísticas destas áreas foram as que mais se assemelharam ao campo úmido amostrado, por apresentarem os maiores valores de índice. 
Tabela 2 - Similaridade florística entre áreas campestres alagadas com o campo úmido da Fazenda Pedra Vermelha, Analândia, São Paulo. ISS: índice de similaridade de Sorensen. *Estudo florístico realizado em parcelas. **Ausência de informação sobre o tamanho da área amostrada. ***Desconsiderou as Lycofitas e Monilófitas.

Table 2 - Floristic similarity between wetlands areas and the moist grassland in Fazenda Pedra Vermelha, Analândia, São Paulo. ISS: Sorensen similarity index. * Floristic study carried out in plots. ** Without information about the size of the sampled area. ${ }^{* * *}$ Studies that discounted the Lycofitas and Monilófitas.

\begin{tabular}{|c|c|c|c|c|c|c|}
\hline Local de estudo & Área (ha) & $\begin{array}{l}\text { Te m p o d e } \\
\text { coleta (meses) }\end{array}$ & $\begin{array}{l}\text { Nú m e ro } \\
\text { de espécie }\end{array}$ & $\begin{array}{l}\text { Número de espécie } \\
\text { em comum }\end{array}$ & (ISS) \% & Referência \\
\hline $\begin{array}{l}\text { ARIE Cerrado Pé-de-Gigante } \\
\text { (Santa Rita do Passa Quatro - SP) }\end{array}$ & 7 & 23 & 150 & 21 & 12,80 & Batalha $1997 * * *$ \\
\hline $\begin{array}{l}\text { Estação Ecológica do Panga - área } 1 \\
\text { (Uberlândia - MG) }\end{array}$ & 5 & 24 & 218 & 31 & 15,57 & Araújo et al. 2002 \\
\hline $\begin{array}{l}\text { Estação Ecológica do Panga - área } 2 \\
\text { (Uberlândia - MG) }\end{array}$ & 12 & 24 & 242 & 38 & 17,61 & Araújo et al. 2002 \\
\hline $\begin{array}{l}\text { Reserva Vegetal do Clube Caça e } \\
\text { Pesca Itororó (Uberlândia - MG) }\end{array}$ & 45 & 24 & 307 & 41 & 16,49 & Araújo et al. 2002 \\
\hline $\begin{array}{l}\text { Margem da estrada de Campo Florido } \\
\text { (Uberlândia - MG) }\end{array}$ & 8 & 24 & 266 & 35 & 15,31 & Araújo et al. 2002 \\
\hline Fazenda da Máquina (Itirapina - SP) & 67 & 46 & 124 & 41 & 27,15 & Tannus \& Assis 2004 \\
\hline $\begin{array}{l}\text { O Parque Estadual do Cerrado } \\
\text { (Jaguariaíva - PR) }\end{array}$ & 24,70 & 28 & 64 & 16 & 13,22 & Linsingen et al. $2006^{* * *}$ \\
\hline Fazenda Água Fria (Alto do Paraíso - GO) & 21 & 14 & 207 & 21 & 10,90 & Munhoz e Felfili (2006a) \\
\hline Fazenda Água Limpa (Distrito Federal) & 16 & 18 & 197 & 20 & 10,66 & Munhoz e Felfili 2007 \\
\hline Parque Estadual do Jalapão (Tocantins) & - & 24 & 136 & 11 & 6,41 & Rezende 2007 \\
\hline $\begin{array}{l}\text { Estação Ecológica de Itirapina } \\
\text { (Itirapina - SP) }\end{array}$ & 56 & 36 & 167 & 57 & 33,04 & Tannus $2007^{*}$ \\
\hline $\begin{array}{l}\text { Unidade de Pesquisa e Desenvolvimento } \\
\text { de Itararé (Itararé - SP) }\end{array}$ & - & 12 & 89 & 35 & 26,21 & Tannus $2007 * *$ \\
\hline Fazenda Água Limpa (Distrito Federal) & 16 & 12 & 221 & 34 & 16,79 & Amaral (2008) \\
\hline Fazenda Pedra Vermelha (Analândia - SP) & 5 & 18 & 178 & - & - & Presente estudo \\
\hline
\end{tabular}

Quando comparado o tamanho da área amostral versus a riqueza de espécies, observase que o campo úmido da Fazenda da Máquina (Tannus \& Assis 2004), da Estação Ecológica de Itirapina (Tannus 2007) e do Parque Estadual do Cerrado (Linsingen et al. 2006) possuem área significativamente maior em relação à área do campo úmido amostrado, contudo relatam a presença de um número menor de espécies. Esses resultados reforçam o conceito estabelecido por Odum (2001), que, embora ocorrente com frequência, nem sempre há uma proporção direta entre o aumento do número de espécie e o aumento da área amostrada.

Para Moreira et al. (2015), as fisionomias de campo úmido e vereda situadas no Brasil Central possuem uma forte similaridade estrutural e florística, principalmente em relação ao estrato herbáceo. Contudo, em se tratando de áreas localizadas no sudeste brasileiro, baseado no Índice de Sorensen entre a área campestre alagada da Fazenda Pedra Vermelha, São Paulo e as quatro veredas situadas no município de Uberlândia, Minas Gerais (Araújo et al. 2002), não foi possível observar-se a existência de uma significativa similaridade florística entre essas formações. Esse resultado permite propor uma investigação aprofunda e detalhada sobre a flora dessas, e de outras áreas campestres alagadas da região Sudeste, a fim de verificar-se, assim como no Brasil Central, é possível diagnosticar uma afinidade florística entre elas. 
Fundamentado nos resultados obtidos através da listagem das espécies ocorrentes no campo úmido da FPV, consta-se o quão pouco se conhece sobre a composição florística e estrutura das formações alagadas. Ressalta-se a relevância e a necessidade de multiplicação de investigações sobre a flora dessas áreas negligenciadas. Além disso, torna-se evidente a importância de se manter o atual status de conservação do campo úmido amostrado, a fim de proteger as espécies ameaçadas, bem como conservar os recursos hídricos e edáficos, além de proporcionar e facilitar meios e incentivos para atividades de pesquisa científica, estudos e monitoramento ambiental.

\section{Agradecimentos}

À Fapesp, a bolsa de Mestrado concedida a NLS (processo 2012/12772-5). Ao MCT/CNPq/ MEC/CAPES/PROTAX (processo 562240/20101), e ao CNPq, a bolsa de produtividade (processo 300240/2009-0), os dois últimos concedidos a JAL. Aos especialistas que auxiliaram na identificação de espécies e aos proprietários da Fazenda Pedra Vermelha.

\section{Referências}

Amaral, A.G. 2008. Mudanças estruturais e florística do estrato herbáceo-arbustivo em um campo sujo e campo limpo úmido na fazenda Água Limpa - DF após um período de sete anos. Dissertação de Mestrado. Universidade de Brasília, Brasília. 180p.

Amorim, P.K. \& Batalha, M.A. 2006. Soil characteristics of a hyperseasonal cerrado compared to a seasonal cerrado and a floodplain grassland: implications for plant community structure. Brazilian Journal of Biology 66: 661-670.

Angiosperm Phylogeny Group (APG). 2009. An update of the Angiosperm Phylogeny Group classification for the orders and families of flowering plants: APG III. Botanical Journal of the Linnean Society 161: 105-121.

Araújo, G.M.; Barbosa, A.A.A.; Arantes, A.A. \& Amaral, A.F. 2002. Composição florística de veredas no Município de Uberlândia, MG. Revista Brasileira de Botânica 25: 475-493.

Batalha, M.A. 1997. Análise da vegetação da ARIE Cerrado Pé-de-Gigante (Santa Rita do Passa Quatro, Goiás). Dissertação de Mestrado. Universidade de São Paulo, São Paulo. 184p.

BFG. 2015. Growing knowledge: an overview of Seed Plant diversity in Brazil. Rodriguésia 66: 1085-1113.

Brito, E.R.; Martins, S.V.; Oliveira-Filho, A.T.; Silva, E. \& Silva, A.F. 2008. Estrutura fitossociológica de um fragmento natural de floresta inundável em área de Campo Sujo, Lagoa da Confusão, Tocantins. Acta Amazônica 38: 379-386.
Coutinho, L.M. 1978. O conceito de cerrado. Revista Brasileira de Botânica 1: 17-23.

Eiten, G. 1983. Classificação da Vegetação do Brasil. CNPq/ Coordenação Editorial, Brasília. 305p.

Eugênio, C.U.O.; Munhoz, C.B.R. \& Felfili, J.M. 2011. Dinâmica temporal do estrato herbáceo-arbustivo de uma área de campo limpo úmido em Alto Paraíso de Goiás, Brasil. Acta Botanica Brasilica 25: 497-507.

Empresa Brasileira de Pesquisa Agropecuária - Embrapa. 2006. Centro Nacional de Pesquisa de Solos. Sistema brasileiro de classificação de solos. Embrapa Produção de Informação, Brasília; Embrapa Solos, Rio de Janeiro. 306p.

Fidalgo, O. \& Bononi, V.L.R. 1984. Técnicas de coleta, preservação e herborização de material botânico. Instituto de Botânica, São Paulo. 62p.

Filgueiras, T.S.; Nogueira, P.E.; Brochado, A.L. \& Guala II, G.F. 1994. Caminhamento - um método expedito para levantamentos florísticos qualitativos. Caderno de Geociência 12: 39-43.

Gonçalves, E.G. \& Lorenzi, H. 2011. Morfologia vegetal: organografia e dicionário ilustrado de morfologia das plantas vasculares. $2^{\mathrm{a}}$ ed. Editora Plantarum, Nova Odessa. 416p.

Guimarães, A.J.M.; Araújo, G.M. \& Corrêa, G.F. 2002. Estrutura fitossociológica em área natural e antropizada de uma vereda em Uberlândia, MG. Acta Botanica Brasilica 16: 317-329.

Ivanauskas, N.M.; Rodrigues, R.R. \& Nave, A.G. 1997. Aspectos ecológicos de um trecho de floresta de brejo em Itatinga, SP: florística, fitossociologia e seletividade de espécies. Revista Brasileira de Botânica 20: 139-153.

Kier, G.; Mutke, J.; Dinerstein, E.; Ricketts, T.H.; Küper, W.; Kreft, H. \& Barthlot, W. 2005. Global patterns of plant diversity and floristic knowledge. Journal of Biogeography 32: 1107-1116.

Kramer, K.U. \& Tryon, R.M. 1990. Introduction to the treatment of pterodophytes. In: Kramer, K.U. \& Green, P.S. (eds.). The families and genera of vascular plants. I. Pteridophytes and Gymnosperms. Springer-Verlag, Berlin. Pp. 12-13.

Linsingen, L.V.; Sonehara, J.S.; Uhlmann, A. \& Cervi, A. 2006. Composição florística do Parque Estadual do Cerrado de Jaguariaíva, Paraná, Brasil. Acta Biológica Paranaense 35: 197-232.

Mamede, M.C.H.; Souza, V.C.; Prado, J.; Barros, F.; Wanderley, M.G.L. \& Rando, J.G. 2007. Livro vermelho das espécies vegetais ameaçadas do estado de São Paulo. Instituto de Botânica, São Paulo. 158p.

Martinelli, G. \& Moraes, M.A. (org.). 2013. Livro vermelho da flora do Brasil. Instituto de Pesquisas Jardim Botânico do Rio de Janeiro, Rio de Janeiro. 1100p.

Meirelles, M.L.; Ferreira, E.A.B. \& Franco, A.C. 2006. Dinâmica sazonal do carbono em campo úmido do Cerrado. Série Documentos, 164. Embrapa Cerrados, Planaltina. 29p. 
Mendonça, R.C.; Felfili J.M.; Walter, B.M.T.; Júnior, M.C.S.; Rezende, A.V.; Filgueiras, T.S.; Nogueira, P.E. \& Fagg, C.W. 2008. Flora vascular do bioma Cerrado: checklist com 12.356 espécies. In: Sano, S.M.; Almeida, S.P. \& Ribeiro, J.F. (eds.). Cerrado: ecologia e flora. Embrapa Informação Tecnológica, Brasília. Pp. 423-1279.

Moreira, S.N.; Eisenlohr, P.V.; Pott, A.; Pott, V.J. \& Oliveira-Filho, A.T. 2015. Similar vegetation structure in protected and non-protected wetlands in Central Brazil: conservation significance. Environmental Conservation 42: 356-362.

Munhoz, C.B.R. \& Felfili, J.M. 2006a. Floristic of the herbaceous and subshrub layer of a moist grassland in the Cerrado biosphere reserve (Alto Paraíso de Goiás), Brazil. Edinburgh Journal of Botany 63: 343-354.

Munhoz, C.B.R. \& Felfili, J.M. 2006b. Fitossociologia do estrato herbáceo-subarbustivo de uma área de campo sujo no Distrito Federal, Brasil. Acta Botanica Brasilica 20: 671-685.

Munhoz, C.B.R. \& Felfili, J.M. 2007. Florística do estrato herbáceo-subarbustivo de um campo limpo úmido em Brasília, Brasil. Biota Neotropica 7: 205-215.

Munhoz, C.B.R. \& Felfili, J.M. 2008. Fitossociologia do estrato herbáceo-subarbustivo em campo limpo úmido no Brasil Central. Acta Botanica Brasilica 22: 905-913.

Munhoz, C.B.R.; Felfili, J.M. \& Rodrigues, C. 2008. Species-environment relationship in the herb-subshrub layer of a moist Savanna site, Federal District, Brazil. Brazilian Journal of Biology 68: 25-35.

Mueller-Dombois, D. \& Ellenberg, H. 1974. Aims and methods of vegetation ecology. Jonh Wiley \& Sons, New York. 547p.

Myers, N.; Mittermeier, R.A.; Mittermeier, C.G.; Fonseca, G.A.B. \& Kent, J. 2000. Biodiversity hotspots for conservation priorities. Nature 430 : 853-858.

Odum, E.P. 2001. Fundamentos de ecologia. Fundação Calouste Gulbekian, Lisboa. 235p.

Oliveira-Filho, A.T. \& Ratter, J.A. 2002. Vegetation physiognomies and woody flora of the Cerrado biome. In: Oliveira, P.S. \& Marquis, R.J. (eds.). The Cerrados of Brazil. Columbia University Press, New York. Pp. 91-120.

Prado, J.; Sylvestre, L.S.; Labiak, P.H.; Windisch, P.G.; Salino, A.; Barros, I.C.L.; Hirai, R.Y.; Almeida, T.E.; Santiago, A.C.P.; Kieling-Rubio, M.A.; Pereira, A.F.N.; Øllgaard, B.; Ramos, C.G.V.; Mickel, J.T.;
Dittrich, V.A.O.; Mynssen, C.M.; Schwartsburd, P.B.; Condack, J.P.S.; Pereira, J.B.S.\& Matos, F.B. 2015. Diversity of ferns and lycophytes in Brazil. Rodriguésia 66: 1073-1083.

Ratter, J.A.; Ribeiro, J.F. \& Bridgewater, S. 1997. The brazilian Cerrado vegetation and threats to its biodiversity. Annals of Botany 80: 223-230.

Reatto, A.; Correia, J.R. \& Spera, S.T. 1998. Solos do bioma Cerrado: aspectos pedológicos. In: Sano, S.M. \& Almeida, S.P. (ed.). Cerrado: ambiente e flora. Embrapa, Planaltina. Pp. 46-86.

Rezende, J.M. 2007. Florística e fitossociologia e a influência do gradiente de umidade do solo em campos limpos úmidos no Parque Estadual de Jalapão, Tocantins. Dissertação de Mestrado. Universidade de Brasília, Brasília. 74p.

Ribeiro, J.F. \& Walter, B.M.T. 2008. As principais fitofisionomias do bioma Cerrado. In: Sano, S.M.; Almeida, S.P. \& Ribeiro, J.F. (eds.). Cerrado: ecologia e flora. Embrapa Informação Tecnológica, Brasília. Pp. 152-212.

Rizzini, C.T. 1963. Nota prévia sobre a divisão fitogeográfica (florístico-sociocológica) do Brasil. Revista Brasileira de Geografia 25: 3-64.

Sarmiento, G. 1992. Adaptive strategies of perennial grasses in South American savanas. Journal of Vegetation Science 3: 325-336.

Scholes, R.J. \& ARCHER, S.R. 1997. Tree-grass interactions in Savanas. Annual Review of Ecology and Systematics 28: 517-544.

Smith, A.R.; Pyer, K.M.; Schuettpelz, E.; Korall, P.; Schneider, H. \& Wolf, P.G. 2006. A classification for extant ferns. Taxon 55: 705-731.

Tannus, J.L.S. \& Assis, M.A. 2004. Composição de espécies vasculares de campo sujo e campo úmido em área de Cerrado, Itirapina, SP, Brasil. Revista Brasileira de Botânica 27: 498-506.

Tannus, J.L.S. 2007. Estudo da vegetação dos campos úmidos do Cerrado: aspectos florísticos e ecológicos. Tese de Doutorado. Universidade Estadual Paulista Júlio de Mesquita Filho, Rio Claro. 147p.

Wanderley, M.G.L.; Shepherd, G.J.; Martins, S.E.; Estrada, T.E.M.D.; Romanini, R.P.; Koch, I.; Pirani, J.R.; Melhem, T.S.; Harley, A.M.G.; Kinoshita, L.S.; Magenta, M.A.G.; Wagner, H.M.L.; Barros, F.; Lohmann, L.G.; Amaral, M.C.E.; Cordeiro, I.; Aragaki, S.; Bianchini, R.S. \& Esteves, G.L. 2011. Checklist das Spermatophyta do Estado de São Paulo, Brasil. Biota Neotropica 11: 193-390. 